\title{
EL CONTROL DE LA INMIGRACIÓN: \\ Política fronteriza, selección del acceso e inmigración irregular
}

\author{
Ana María López Sala \\ Departamento de Sociología \\ Universidad de La Laguna
}

\begin{abstract}
This article describes and compares immigration control policies and their outcomes in different countries and migration systems in the last decades. In the three decades after the Second World War migration and refugees issues received little attention in public debates. The quest to control migration flows has in many senses proved elusive. Although governments have been keen to demonstrate their capacity to limit the number of immigrants, several domestic and international factors have combined to frustrate their attempts.
\end{abstract}

KEY WORDS Immigration policy, comparative migration control, borders and migration, irregular migration, prevention and admission policies.
RESUMEN Este artículo describe y compara las políticas de control de flujos diseñadas e implantadas en distintos paises y sistemas migratorios a lo largo de las dos últimas décadas. La entrada del control de la inmigración en la agenda politica de los paises receptores es relativamente reciente. Hasta los años setenta la inmigración permanece al margen de los debates públicos. La búsqueda de procedimientos eficaces de control se ha tornado una tarea inaprensible $y$, aunque los gobiernos han intentado mostrar su capacidad para controlar los influjos, diversos factores de naturaleza nacional e internacional han moldeado sus respuestas y frenado su eficacia.

PALABRAS CLAVE Politica migratoria comparada, control de flujos, inmigración irregular, políticas preventivas y de admisión de la inmigración, fronteras.

sino también a la variedad de aspectos que comporta el desarrollo de las políticas migratorias. Se trata de un tipo de acción en donde confluyen múltiples intereses y asuntos públicos, que ha llegado a convertirse en algunos paises y en diversos niveles de gobierno en objeto de intervención transversal y en la que concurren decisiones adoptadas en la arena política regional, doméstica e internacional. Este enfoque incorpora en los últimos años otros objetos de indagación como los lazos entre inmigración y ciudadanía, la acción colectiva y la movilización de intereses de los inmigrantes, la asignación de derechos a las minorias socioculturales y la conformación de formas de identidad e instituciones transnacionales.

Diversos factores explican este interés académico en el estudio del gobierno de la migración. Hasta la segunda mitad del siglo XX, permanece como un tema de escasa relevancia politica. No es nueva, sin embargo, su regulación que fue practicada con mayor o menor intensidad a lo largo del último siglo. En los años ochenta asistimos, sin embargo, a un paulatino y, por el momento, irreversible proceso de politización de la cuestión migratoria. La aceleración y diversificación de los flujos ha planteado la necesidad de hacer frente a la inmigración como un fenómeno profundamente heterogéneo que exige políticas adaptadas a su variedad en

La complejidad de esta nueva perspectiva en el análisis de las migraciones internacionales se debe no sólo a la novedad, 
términos de composición y proyecto. La intensificación de las corrientes de refugiados y menores ha incorporado estas iniciativas a la agenda de organizaciones internacionales y de derechos humanos. Su impacto y complejidad demográfica ha inspirado, asimismo, nuevas medidas en áreas como la sanidad, la educación y la formación no reglada y ha activado la creación y la cooperación institucional en los nuevos paises receptores. El mantenimiento de la demanda de mano de obra ha necesitado de acciones dirigidas al suministro temporal y permanente de trabajadores. La instalación definitiva y el aumento de las reclamaciones ligadas a derechos lingüisticos y religiosos han moldeado formas de integración más permeables a la reproducción cultural de las comunidades y al acceso a derechos ciudadanos a la par que medidas más eficaces de lucha contra la inmigración irregular.

Paulatinamente se han conformado procedimientos de regulación de la entrada y del establecimiento en los países de acogida, en donde se vierten propuestas sobre la idoneidad del número y las caracteristicas sociodemográficas, nacionales y culturales de los candidatos a la migración. Es bien conocido hoy que durante décadas las migraciones procedentes de paises asiáticos, el Sur de Europa, el Caribe y los paises eslavos fueron prohibidas o fuertemente restringidas en los Estados receptores a partir de la influencia política de movimientos nativistas y de entendimientos sociales sobre el carácter inasimilable de ciertos tipos de inmigrantes. En comparación con las cada vez más complejas políticas actuales, las acciones de las décadas centrales del siglo XX resultan parciales en el alcance de sus objetivos, escasas en el número de paises que las practicaban y modestas en términos de los recursos que empleaban (Monar, 1997). Las politicas migratorias siguen determinando en cierta medida la cantidad y composición de los candidatos a la migración, lo que hemos denominado las condiciones de elegibilidad de los migrantes (López Sala, 2005b), pero no se detienen en la entrada, sino que incorporan a sus objetivos la gestión de los efectos de la residencia y del establecimiento. La complejidad de la politica migratoria se compagina con su carácter cambiante de acuerdo con la volatilidad del fenómeno y las condiciones coyunturales de los paises receptores. De ahi su permanencia en la agenda política, -como pone de manifiesto el insistente recurso a este aspecto en los programas electorales- y su condición de "bien perecedero", sometida a constante evaluación crítica (véase Arango y Sandell, 2004).

Resulta demasiado ambicioso para un artículo de estas dimensiones realizar un análisis comparado de políticas migratorias y destilar una tipología si atendemos a dos aspectos que las dotan de gran complejidad estructural: las esferas de la intervención y la geografía de esta política en los diversos sistemas migratorios. Por política migratoria puede entenderse, grosso modo, el conjunto de mecanismos legales y administrativos articulados por lo general desde el Estado, pero también desde otras instituciones supranacionales, que regulan el acceso al territorio, la estancia y el establecimiento, la integración socioeconómica y cívica de los inmigrantes y el disfrute de derechos ciudadanos, asi como la incorporación a la comunidad política. Habría que considerar, por añadidura, como esferas específicas, la regulación de la entrada al mercado de trabajo en destino y la implantación de iniciativas económicas en las cuencas migratorias orientadas a contener los flujos de salida ${ }^{1}$. Estas esferas -control de flujos y control interno, integración, cooperación al desarrollo y política de naturalización- han servido de referencia a la hora de establecer pautas y de construir modelos. No debemos olvidar, sin embargo, que el peso de cada una ellas en el conjunto de la política ha sido muy variado. En la actualidad las acciones de regulación y de integración comparten protagonismo y concentran una buena parte de los recursos, en especial las medidas dirigidas al control fronterizo y a la lucha contra la inmigración irregular. Por ello este artículo se centrará en estos dos últimos aspectos -en el terreno de la immigration policy como se denomina en la literatura anglosajona- a la espera de que otros formatos permitan objetivos más ambiciosos y respondiendo a la orientación descriptiva que se demandó a la autora de cara a la presente publicación. Las conclusiones, por tanto, no tienen pretensión de exhaustividad. Tan sólo apuntan algunos rasgos parciales que han sido explorados con mayor profundidad en otros artículos (López Sala, 2003, 2005c).

En sentido estricto, estas diversas esferas no distinguen entre tipos de migrantes, por lo que las acciones dirigidas a las migraciones forzosas, las políticas de refugio y las de atención a los desplazados, no conforman una parte en sí misma, a pesar de la existencia de una regulación propia en el ámbito nacional e internacional. Algunos regimenes supranacionales de extranjería, como el erigido en el seno de la Unión Europea, merecen, asimismo, un análisis específico, al tratarse de sistemas de regulación paralelos a los generales. El comunitario, por ejemplo, es un sistema de regulación débil que incorpora la libertad de entrada y establecimiento, el acceso al mercado de trabajo en igualdad de condiciones que los nacionales ${ }^{2}$, amplios derechos económicos y sociales, asi como el sufragio en las elecciones locales y autonómicas. 
En términos generales puede ser también descrito como un régimen indirecto de acceso privilegiado a la ciudadania a través de una regulación privilegiada de la residencia en paridad con los nacionales.

La segunda dificultad que entraña el análisis comparado de las politicas migratorias responde a la diversidad de formas que adopta en los paises receptores. Esta variedad es resultado de la combinación de factores de gran calado: la tradición receptora, las diferentes experiencias históricas sobre la conformación de los Estados nacionales liberales y la ciudadanía democrática, el grado interno de diversidad cultural y social, asi como la extensión y características del Estado social. Las diferencias existentes entre las sociedades occidentales y otras regiones del globo en cuanto a estas variables dificultan extremadamente los estudios comparados, tanto de casos como de variables, una tarea que ha sido emprendida por el momento con extremada cautela (véase Castles, 1998, 2001). De ahi que, pese a que ello implique una visión extremadamente reducida si tenemos en cuenta la dimensión universal de las corrientes migratorias contemporáneas, este artículo se centre especialmente en el sistema migratorio europeo y norteamericano, aunque se hará referencia someramente a otras áreas geográficas implicadas en la movilidad internacional.

Es en la Europa receptora y en los paises anglosajones de larga tradición de acogida, como Australia, Nueva Zelanda, Estados Unidos y Canadá, donde estas políticas han adquirido mayor complejidad y desarrollo. También en estos Estados se han realizado significativos esfuerzos dirigidos a la evaluación de las políticas implantadas -a través, entre otras fórmulas, de la elaboración de catálogos de buenas y malas prácticas- y a la obtención de buenos registros del fenómeno que se dirigen a conseguir ajustados diagnósticos. El cumplimiento de los principios que emanan del derecho internacional y la salvaguardia de derechos fundamentales en los cruces fronterizos y en la instalación han sido objeto en ellos de mayor control democrático y social. El reconocimiento de derechos ciudadanos a las comunidades inmigrantes es extremadamente débil o inexistente en regímenes teocráticos o autoritarios como los observados en los sistemas migratorios del Golfo Pérsico y del continente africano que tienen, en términos de volumen, un peso destacado como áreas receptoras (Martin y Widgren, 2002; Adepoju, 2000, 2001). La ausencia de garantías en el disfrute de derechos fundamentales a los inmigrantes temporales e instalados se extiende a un buen número de paises asiáticos que han experimentado recientemente su transición migratoria como Malasia o los dragones asiáticos en donde, por añadidura, las políticas de integración y de naturalización han tenido un desarrollo parcial y restrictivo.

\section{Las políticas de regulación de los flujos migratorios.}

\subsection{La similitud de las medidas de control fronterizo}

Las politicas migratorias iniciales surgieron con el intento de regular la dimensión y caracteristicas de los influjos de trabajadores. La articulación de vías de selección étnica impregnó los primeros ordenamientos a la par que se construian procedimientos de control de las entradas a través de la creación de documentos de identificación y cruce, como los pasaportes y el visado. La dimensión cualitativa de la inmigración se combina con la selección del volumen desde los años setenta. Pero el desequilibrio entre la oferta y la demanda migratoria y el desajuste entre el reconocimiento de la libertad emigratoria en el derecho internacional y el sometimiento del derecho a inmigrar a la soberanía de los paises de acogida ha creado un modelo inestable en donde los Estados receptores deben invertir ingentes cantidades de recursos para hacer frente a este desequilibrio a través de acciones políticas. A lo largo de las últimas tres décadas la mayor parte de los Estados occidentales han desarrollado medidas dirigidas a la contención de las corrientes migratorias a través de la aprobación de leyes, el desarrollo de las agencias e instituciones especializadas y el desvío de gran cantidad de fondos públicos. Por mencionar algunos datos recientes, en el año 2003, Estados Unidos, Canadá, Alemania, Gran Bretaña y Holanda invirtieron 17.000 millones de dólares en hacer efectiva su política de control migratorio. Esta cantidad representaba casi dos tercios del total de la ayuda oficial que destinaban a la cooperación al desarrollo (véase Martin, 2003). La inversión ha sido especialmente abultada en los nuevos paises de inmigración del sur europeo convertidos con Schengen en Estados-guardián de las fronteras de la Unión. En el caso español, la implantación del Sistema Intensivo de Vigilancia Exterior en el Estrecho y en las provincias orientales del archipiélago canario ha supuesto, por ejemplo, una inversión de 106 millones de euros entre el año 2002 y el 2004 y las previsiones son que entre el 2005 y el 2008 se invertirán 130 millones más.

Las medidas adoptadas en esta materia en los diversos sistemas migratorios han sido muy similares desde mediados 
de los ochenta. El fortalecimiento de las fronteras, la aplicación de tecnología de última generación y la colaboración multilateral se han ampliado con el uso de mecanismos de control previo y de "externalización" del control (Boswell, 2003b) a partir de las concesiones de visado, la firma de acuerdos de readmisión de solicitantes de asilo y el establecimiento de sanciones a las compañias de transporte de pasajeros que convierten en la práctica a los profesionales del trasporte en funcionarios de frontera. Sin embargo, a pesar de la analogía de la acción y del consenso existente en las administraciones y la opinión pública acerca de la necesidad de controlar los flujos de personas, las asociaciones étnicas y de defensa de los derechos humanos abogan por desarrollar fórmulas que permitan canales legales de entrada y de establecimiento, así como medidas especiales que faciliten la reagrupación familiar y la protección de refugiados y menores. El elemento de discusión descansa en la cautela señalada por una parte de la sociedad civil de que el refuerzo de las fronteras no puede realizarse a expensas de derechos fundamentales. Una línea de argumentación paralela ha surgido en esta materia en el seno de la filosofía política, en donde, se sostiene que el principio de justicia establece los parámetros legalmente legítimos de la práctica política. Las aportaciones, aunque todavía escasas, subrayan que el desarrollo de una teoria de la justicia que incorpore la inmigración debe basarse en el principio de igualdad y de no-discriminación según criterios raciales y económicos (véase Carens, 1996, 1997, 2002; Ingram, 2002 y Zapata, 2001), variables que en mayor o menor medida han moldeado hasta la fecha las respuestas estatales.

El desarrollo de políticas complejas de control se acompaña de la evidencia de la creciente dificultad de los gobiernos para intervenir con éxito en los procesos de regulación y vigilancia fronteriza. De hecho, uno de los ensayos recientes más influyentes sobre este tema se apoya precisamente en estos dos aspectos para caracterizar el estado del control migratorio. En este trabajo se enuncia, por un lado, la tesis de la convergencia según la cuál existe una creciente similitud entre las políticas de control de flujos empleadas en los paises más industrializados, lo que ha llevado a la desaparición de las diferencias tradicionales entre los paises clásicos de inmigración y los europeos que se convierten en Estados receptores después de la Segunda Guerra Mundial. La tesis del desajuste indica que la distancia entre los objetivos de las políticas de control y los resultados es amplia y creciente, lo que ha provocado un clima de opinión hostil a la inmigración y un aumento de la presión sobre los gobiernos para que adopten medidas aún más restrictivas (Cornelius, Martin, y Hollifield, 1995; Martínez y Moualhi, 2005) ${ }^{3}$.

Los paises receptores rara vez han puesto en funcionamiento políticas de inmigración "cero». Por ello es más adecuado hablar de la implantación en los noventa de medidas de acceso condicionado y restrictivo, donde se ha impulsado la entrada y el establecimiento de ciertas categorias de inmigrantes y se han puesto serios impedimentos a otro tipo de flujos. Existe de hecho hoy cierto acuerdo entre los especialistas a la hora de sostener que la retórica de la política de inmigración cero ha sido una falacia y que se han mantenido abiertas vías que han permitido la llegada de familiares y de trabajadores, de forma explícita o encubierta, para hacer frente a las necesidades de los mercados laborales nacionales ${ }^{4}$. De ahi, la defensa de la tesis de la ambivalencia de los Estados respecto a la inmigración irregular (véase Cornelius, 2000). Las políticas de control de los flujos migratorios pueden ser calificadas hoy, por tanto, como políticas de acceso selectivo que a partir de la acción unilateral del Estado determinan de forma indirecta las condiciones de "elegibilidad" de los candidatos a la inmigración y condenan a ciertas categorías de inmigrantes a la clandestinidad (Massey y Durand, 2003).

Recientemente ha aparecido en algunos paises de Europa, un discurso todavia muy minoritario, pero que, sin duda, ha alimentado el debate y la discusión, que se aleja del talante restriccionista de los años ochenta y noventa. La aparición de nuevas tendencias económicas internacionales, la transformación de los mercados de trabajo nacionales y de la estructura demográfica marcan la urgencia, indican, por diseñar nuevos modelos de admisión. Esta demanda se centra, en especial, en políticas que permitan el reclutamiento de trabajadores altamente cualificados y de mano de obra temporal y permanente para cubrir vacantes en ciertos nichos laborales: entre otros en el sector servicios, la hostelería y la agricultura en los paises del sur de Europa y Estados Unidos y la sanidad y la educación en paises europeos de tradición receptora como Gran Bretaña o Alemania. El argumento demográfico vinculado al envejecimiento de la población y la quiebra del sistema de pensiones ha adquirido un peso destacado en el debate, en particular desde el polémico informe del año 2000 de las Naciones Unidas. El control de flujos se ha configurado, en definitiva, como una esfera de la política pública sometidas a una doble tensión, a la búsqueda de un adecuado equilibrio entre las fuerzas

ARBOR CLXXXI 713 MAYO-JUNIO [2005] 27-39 ISSN: 0210-1963 
que consideran idóneo el cierre y, por otro lado, las presiones de muy diversa naturaleza, que las dirigen a la apertura.

Tanto en el caso europeo como en el norteamericano se aprecia una creciente ansiedad en la opinión pública en torno a la idea de la porosidad y vulnerabilidad de las fronteras y la tesis de la invasión migratoria. Catherine Whitol de Wenden mantiene, por ejemplo, en el caso francés, que la politización de la cuestión migratoria durante los años ochenta y noventa ha hecho que las decisiones se vean cada vez más sometidas a los movimientos de opinión que a una politica migratoria concertada con todas las partes interesadas (Whitol de Wenden, 1992, 2000). Su conclusión es que el creciente papel de la opinión pública, convertida en el control del control, lleva a los Estados a mostrar firmeza en el discurso público, aún llevando a cabo una política subterránea de apertura de fronteras. Similares conclusiones se obtienen en los estudios sobre la evolución de las políticas migratorias en Italia, Alemania y Gran Bretaña a lo largo de las dos últimas décadas. Cristina Boswell sostiene, a modo de síntesis, que en la política migratoria europea se observa lo que ha calificado como "un desajuste populista»: un desajuste entre las necesidades económicas y las medidas factibles que pueden elaborar los Estados para restringir la inmigración y las presiones de la opinión pública y de los partidos anti-inmigración. El resultado del enfrentamiento entre estas fuerzas ha sido, por el momento, la aplicación de medidas poco realistas y parcialmente ajustadas a la demanda del mercado de trabajo.

\subsection{La lucha contra los flujos no autorizados}

La lucha contra los flujos no autorizados de trabajadores forma parte de la agenda política nacional y europea en materia de inmigración. El marco de colaboración multinacional que crearon los acuerdos de Schengen y de Dublín estableció vias de cooperación que tras la firma del Tratado de Amsterdan se transforman en parte integrante del primer pilar. La lucha contra las redes que trafican con personas y la inmigración clandestina han estimulado durante la segunda mitad de los años noventa, múltiples declaraciones en los órganos de las Naciones Unidas y en las instituciones europeas, así como el estudio y asesoramiento en materia de intervención política a través de organizaciones como la OIT y la IOM. La declaración de Bangkok y los recientes protocolos sobre tráfico de personas y de migrantes de la nueva Convención Internacional contra las redes transnacionales de crimen organizado establecen, por ejemplo, mecanismos legales de gobierno de la migración en el contexto de la supresión del crimen, la prevención y el régimen sancionador. En el año 2000 se estimaba que el tráfico de personas se había convertido en un negocio tan lucrativo como el tráfico de estupefacientes, pero menos arriesgado si atendemos a la tipificación y gravedad de las penas. No debemos olvidar, sin embargo, que la identificación entre inmigración irregular y delincuencia es una estereotipo que responde a la profunda estigmatización que los inmigrantes clandestinos y los solicitantes de asilo han sufrido a lo largo de los noventa. La opinión pública les señala como causantes de la criminalidad creciente y de la inseguridad ciudadana y se presentan frecuentemente como un peligro para la identidad nacional y la seguridad.

La inquietud pública hacia la inmigración irregular, ha inspirado un gran número de iniciativas que incluyen el blindaje fronterizo, el aumento del personal en operaciones de patrullaje y la creación de cuerpos especiales en la administración y la policía dedicados al control. El empleo de tecnología militar en la vigilancia aduanera y fronteriza ha llevado a algunos autores a hablar de estado de alarma, de excepción migratoria y de militarización de la frontera ${ }^{5}$. Este es el caso de las medidas adoptadas en la frontera entre México y Estados Unidos a lo largo de los noventa a partir del lema, "prevention through deterrence" 6 y de las sucesivas operaciones denominadas Blockade 7 en El Paso, Texas (1993), Gatekeeper en San Diego-Tijuana (1994)8, Safeguard en Nogales, Arizona (1994) y Río Grande en el sur de Texas (1997) (véase Alba, 1999). Este tipo de medidas, a pesar de su dudosa efectividad, han asegurado, indica Aleinikoff, la integridad simbólica de la frontera, generando una imagen seductora de control estatal y reforzando el mito de que la respuesta al problema de la inmigración irregular se encuentra en las acciones fronterizas, mientras se oscurece la incómoda realidad de la existencia de un mercado de trabajo informal transnacional (véase Aleinikoff, 2002; Andreas, 1998 y 2000). Las dudas sobre la efectividad del Estado para cumplir la misión de asegurar la uintegridad territorial» han activado el desarrollo de patrullas civiles en los estados fronterizos de Arizona y Texas dedicadas a la tarea de vigilar e informar de la presencia de inmigrantes clandestinos. En los últimos meses han sido frecuentes las noticias de la intensificación de esta actividad en Arizona a través del denominado Proyecto Minuteman ${ }^{9}$.

Los atentados del 11 de septiembre han impelido mayor fuerza al nexo entre seguridad e inmigración. El diseño 
político se ve impregnado por los objetivos de permitir y promocionar la llegada de aquellos considerados adecuados para la seguridad nacional y ha tenido la secuela de estigmatizar a las comunidades establecidas consideradas potencialmente peligrosas, en especial, a las minorias musulmanas. A los presuntos vínculos entre migración y tráfico de drogas, de armas y de personas se añade en los últimos años la relación entre inmigración y terrorismo internacional. El resultado ha sido lo que ha sido calificado por Faist como una "securization" de la inmigración. El objetivo de la lucha contra el terrorismo debía incorporarse, han afirmado los responsables políticos, a las políticas migratorias. Los efectos de este nuevo clima no se dejaron esperar con medidas tales como la aprobación de nuevos documentos de identificación más difíciles de falsificar, mayores facilidades para detener en frontera o en el interior del territorio a inmigrantes susceptibles de pertenecer o ser simpatizantes de organizaciones que tienen vínculos con el terrorismo -a través de la Patriot Act en EEUU o de las leyes antiterroristas aprobadas en Gran Bretaña y Canadá- la suspensión temporal de los programas de reasentamiento de refugiados en Estados Unidos y Australia, el incremento de los recursos dirigidos a las deportaciones y a la revisión de las solicitudes de asilo y la posibilidad de solicitar listados de pasajeros a las compañías aéreas (véase Zolberg, 2002).

Otro tipo de medidas de regulación migratoria han intentando, en palabras de los responsables políticos, contener el efecto de atracción que los sistemas del bienestar producen sobre los candidatos a la migración. El argumento de la quiebra de este sistema como consecuencia de la presión ejercida por los inmigrantes ha devenido en reformas legales que limitan seriamente su acceso, en especial de la inmigración no autorizada, a los servicios sociales públicos. Una de las actuaciones de mayor impacto político en la segunda mitad de los noventa fue la aprobación en el estado de California de la ley conocida como Proposición 187. Esta ley, cuyo objetivo fue restringir los derechos sociales de los inmigrantes irregulares ${ }^{10}$, tuvo como finalidad última limitar la instalación de inmigrantes en este estado ampliando el control de la inmigración de las fronteras al interior del territorio. También en Europa se han introducido medidas de exclusión de los sistemas de seguridad social y de los servicios públicos de los inmigrantes irregulares como vías de disuasión desde el origen. En la práctica, sin embargo, se aprecia un suministro "informal» de prestaciones sociales al margen de las que realizan las agencias gubernamentales a través, en especial, de la sociedad civil (Hammar,
1999; Zincone, 1999). Las reformas han afectado, por añadidura, a un derecho básico, como el derecho a la vida familiar, a través de las modificaciones en la gestión de la reagrupación. Los medios han sido no tanto la reducción de los familiares susceptibles de ser reagrupados, como el incremento de la exigencia de recursos económicos por parte del inmigrante residente. Las limitaciones han llegado a afectar seriamente a los sectores más desfavorecidos no sólo de los inmigrantes residentes, sino incluso de los ciudadanos con familiares en el extranjero.

El objetivo del control cuantitativo en el que se inspira la regulación migratoria entra en colisión con los efectos que la acción fronteriza tiene sobre la creciente vulnerabilidad de los migrantes irregulares, en concreto, entre sectores especialmente frágiles como los solicitantes de asilo, las mujeres y los menores de edad. La integridad física y el derecho a la vida de estos colectivos permanecen en segundo plano en el diseño político, aunque en muchas ocasiones los migrantes se convierten en victimas de las mafias $y$, de forma indirecta, del blindaje fronterizo. La densidad fronteriza acrecienta no sólo los costes económicos del cru-

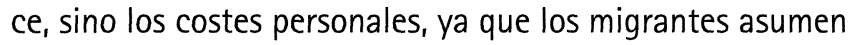
nuevos riesgos para eludir el apresamiento atravesando terrenos desérticos, plagados de animales salvajes o realizando largas travesias a través del mar en barcas mal equipadas y sobrecargadas ${ }^{11}$.

\section{La selección de los trabajadores: nacionalidad y credenciales.}

Las políticas de regulación de flujos se han convertido en una esfera de intervención ambivalente en el terreno de la selección nacional de los migrantes. La creciente diversidad cultural de las nuevas y viejas sociedades de acogida ha desatado todo tipo de temores entre la población y los líderes de opinión. Son muchas las voces que sostienen que la presencia de extranjeros y la formación de minorias étnicas pueden llegar a transformar la identidad nacional, la idiosincrasia cultural de las sociedades receptoras y socavar la democracia al apoyar una cultura política contraria a los principios que emanan del liberalismo. Otros teóricos más alarmistas, además de señalar las inquietantes consecuencias que la inmigración puede provocar en los paises europeos y en Norteamérica, plantean las enormes dificultades de la incorporación de ciertas comunidades a la sociedad general, ejemplificado en el caso de los hispanos en Estados Unidos y los musulmanes en Europa. Aparecen de nuevo

ARBOR CLXXXI 713 MAYO-JUNIO [2005] 27-39 ISSN: 0210-1963 
en primer plano algunas viejas ideas acerca de la condición "inasimilable" de ciertas categorias de inmigrantes y el temor ante la conformación de sociedades paralelas divididas a lo largo de identidades mutuamente excluyentes con escasa cohesión social (véase Sartori, 2001; Huntington, $2004)^{12}$. Por el momento seria demasiado arriesgado afirmar que las políticas de inmigración han recuperado en las sociedades occidentales los tonos selectivos en términos raciales que las caracterizaron en las últimas décadas del siglo XIX y las primeras del XX (Gabbacia, 1999). Sin embargo, existen algunos indicios que revelan la promoción, a través de medidas de naturaleza administrativa, de la entrada y el establecimiento de ciertas categorías de extranjeros consideradas más proclives a integrarse en la sociedad receptora, por criterios como la lengua o la religión. En el caso español, por ejemplo, se ha sostenido, que a finales de la década pasada se empezó a poner en práctica una politica que promovió la llegada y el establecimiento de ciudadanos latinoamericanos y europeos del este frente a los flujos procedentes de Marruecos, de ahi la denominación de los hispanoamericanos como los inmigrantes preferidos (véase Izquierdo, López y Martínez, 2002). La selección nacional, étnica y religiosa es mucho más acentuada como variable de acceso en otros paises receptores como Israel o Japón.

Los argumentos sobre la necesidad de mantener los Estados del bienestar han sido utilizados para apoyar cierto tipo de flujos, en especial, los de trabajadores jóvenes de alta cualificación. Por el contrario, la tesis de la limitación de los recursos públicos disponibles ha sido empleada para justificar medidas que impidan los flujos no requeridos. Geddes ha resumido perfectamente en un artículo reciente los lazos entre migración y Estado social en la construcción de las politicas públicas. Aparecen por un lado, indica, presiones para demarcar más restrictivamente la comunidad de los receptores legítimos de los beneficios públicos y se establecen medios para mantener fuera de esa comunidad a los sujetos de formas de migración que se consideran abusivas o contraproducentes para el interés general, a la par que se promueven las corrientes que pueden realizar una clara contribución a los paises receptores (Geddes, 2003). El resultado es una selección que determina el contorno de los elegidos a poder establecerse por criterios como la capacidad de inversión o la capacitación profesional.

La principal diferencia observada en la reciente regulación migratoria entre los países clásicos de inmigración y los países europeos, es el mantenimiento en los primeros de planes de inmigración permanente, un tipo de programas que apenas han sido desarrollados en nuestro continente. Los flujos permanentes han estado muy vinculados en los primeros al establecimiento de complejas políticas de admisión en donde se combinan criterios de distinta naturaleza y que en la práctica funcionan como un sistema de elegibilidad por puntos. Tras desmantelar las politicas de inmigración racialmente selectivas, los vínculos familiares con ciudadanos y residentes permanentes, las razones humanitarias, la cualificación y los conocimientos lingüísticos se convirtieron en criterios de peso a la hora de la admisión. Australia, por ejemplo, ha aplicado un sistema que combina el reclutamiento de familiares ( a través del estatuto denominado "preferencial» o "concesional" dependiendo de la proximidad de la filiación), los credenciales educativos ${ }^{13}$ y las causas humanitarias, en donde se distingue entre refugiados, perseguidos y desplazados por desastres naturales. Tanto en Australia como en Canadá, la cualificación profesional y la educación (reglada, formación especializada y lingüistica) han tenido en la última década un papel sobresaliente en los procesos de selección. Los especialistas han indicado que el uso de estos criterios en la admisión proporciona una doble ventaja: asegura por un lado, una alta productividad de los migrantes $y$, por otro, reduce los costes educativos de los paises receptores ya que los gastos de formación se realizan en los paises de origen. Los criterios familiares y humanitarios han moldeado el sistema general de admisión de los inmigrantes en Estados Unidos, aunque la cualificación ha sido el criterio clave para la entrada de los denominados migrantes independientes que constituyen una categoria especifica.

Las políticas de control de flujos han sido moldeadas en las últimas décadas también por la percepción de la inmigración como un recurso económico, que ha tenido un especial impacto en el diseño de programas de reclutamiento de trabajadores de alta cualificación y de inversores ( dentro de los programas denominados de Business Migration) que se han desarrollado en Australia, Canadá y Estados Unidos, pero que están empezando a aplicarse también en Alemania y Gran Bretaña. Alemania introdujo, por ejemplo, en el 2000 un sistema dirigido a especialistas en tecnologias de la información y de la comunicación denominado Carta Verde. La previsión de escasez nacional ha autorizado la contratación de 10.000 trabajadores que pueden permanecer en el pais durante 5 años e instalarse con sus familias. Este programa no permite, sin embargo, la solicitud posterior de permisos de trabajo permanentes. En Gran Bretaña 
se implantó a finales del 2002 un plan dirigido a trabajadores de alta cualificación que quisieran entrar en suelo británico para buscar empleo. La admisión se regula a través de un sistema de puntos en donde los criterios fundamentales son el nivel de formación, la experiencia laboral previa, los ingresos pasados y los logros alcanzados en la profesión. La admisión permite el acceso posterior a un permiso de residencia permanente siempre que el trabajador se haya insertado "satisfactoriamente" en la economía británica.Las barreras a la entrada han sido reducidas, en definitiva, en aquellos casos en los que los inmigrantes potenciales cuen$\tan$ con credenciales educativos demandados. $Y$ es que cada vez resulta más evidente la competencia entre Estados por conseguir mano de obra de elevada cualificación para algunos sectores considerados clave en el desarrollo de la economía, de ahí la extensión reciente de este tipo de medidas a algunos países europeos y asiáticos que llevan décadas reclutando trabajadores manuales bajo el objetivo de atraer a los «inmigrantes adecuados».

Algunos de estos programas están siendo implantados tímidamente en otras economías cada vez más fuertes como en el caso de Japón, Singapur, Malasia y Taiwán (véase Alarcón, 2000, 2001). El control de los flujos migratorios ha adquirido un gran protagonismo en la última década en Japón y en los dragones asiáticos, que experimentaron su transición migratoria en los años setenta. La aparición de una economía deficitaria en mano de obra se combinó con una regulación migratoria débil, a partir de la negación de la condición receptora de estos paises. Esta regulación débil se transforma en férreo control a principios de los noventa. La consideración de la inmigración como un fenómeno temporal y el mantenimiento de una elevada demanda han conformado un sistema de reclutamiento temporal de trabajadores extranjeros que persigue la rotación continuada en un esquema similar, en cierto sentido, al modelo gastarbeiter diseñado en la Europa de los años sesenta. De hecho han sido muchos los paralelismos que se han establecido entre la situación en estos paises asiáticos y la Europa anterior a 1973 (Skeldon, 2000), pero como es bien sabido no hay nada tan permanente como un inmigrante temporal (Hugo, 2003). La deficiencia de las políticas desarrolladas para hacer frente a las necesidades de la economía y la estrechez de las vías de establecimiento han nutrido la inmigración irregular.

En Japón el reclutamiento de trabajadores se inicia en los años setenta con el establecimiento de visados para mujeres dedicadas a la industria del entretenimiento, una forma encubierta de referirse a la industria de los servicios sexuales. En los noventa la dimensión de esta clase de flujo alcanza la cifra de los 50.000 permisos anuales, pero algunas investigaciones indican que el volumen real triplica este número (véase IOM, 1997). A lo largo de los ochenta y los noventa han crecido enormemente las migraciones de trabajadores manuales que se emplean en los sectores descritos en japonés con la letra K: kitanai, kiken y kitsui ${ }^{14}$. La maduración del fenómeno ha producido algunas transformaciones en la inserción laboral y cambios de gran calado en la realidad social japonesa. Se aprecia, por ejemplo, una tendencia clara hacia la diversificación laboral de los migrantes en otros sectores de los servicios, la industria y la construcción, mientras que la llegada de trabajadores de alta cualificación se realiza, por lo general, a través de acuerdos privados concertados con las multinacionales y apoyados por el Estado. Por añadidura la paulatina conformación de familias y de comunidades extranjeras puede suponer en las próximas décadas la aparición de una nueva sociedad multicultural, un acontecimiento inaudito en la historia moderna de Japón (Gungwu, 1993; Fukuoka, 2000).

El diseño de las políticas de inmigración y la selección de trabajadores en Japón se ha articulado a través de la persistencia de varios mitos: el de Japón como un país sin historia receptora, la idea de homogeneidad racial de la nación japonesa que ha devenido en un entendimiento étnico y esencialista de la comunidad nacional y la creencia en la eficacia del Estado para prevenir la inmigración no deseada (véase Douglass y Roberts, 2000; Goodman, Peach, Takenaka y White, 2003). Estos factores han tenido un impacto en las politicas de acceso condicionado que han adoptado en los noventa un carácter selectivo a través de la promoción de la llegada de nikkeijin ${ }^{15}$ y el cierre de los canales de entrada de trabajadores manuales. El control de flujos, en definitiva, responde, por tanto, a la asunción de que los extranjeros no pueden incorporarse a la sociedad japonesa bajo la premisa de que esta es una comunidad «racial y cultural» única. El resto de los trabajadores admitidos, al margen de su cualificación, son considerados temporales, con un proyecto de retorno. Las necesidades de trabajadores manuales y de técnicos medios son cubiertas, indican las autoridades, a través de los programas de establecimiento de nikkeijin (Miyoshi, 2003).

A finales de los años noventa los dragones asiáticos presentaban un sistema de regulación migratoria sin tintes selectivos en términos raciales. La cualificación se ha convertido 
en el factor determinante de la admisión. El régimen migratorio confiere un estatuto temporal a los trabajadores extranjeros con derechos muy limitados. La escasez de derechos sociales y laborales se acompaña de una aguda restricción -en ocasiones puede ser calificada de prohibiciónde la reagrupación familiar, sólo permitida en el caso de los migrantes cualificados (Piper, 2004). Este sistema se acompaña de rígidas medidas de prevención de los flujos no autorizados, politicas de visados muy restrictivas y la implantación de medidas legales que incluyen graves sanciones para los inmigrantes irregulares y sus empleadores ${ }^{16}$. Sin embargo, es posible también aplicar en el caso de estos nuevos paises de recepción, la tesis del desajuste. Dos son los factores destacados como significativos a la hora de explicar la pérdida de control de los gobiernos sobre los flujos de entrada. En primer lugar el papel de las redes migratorias, como se ha observado en el caso de Europa y Norteamérica. Existe, sin embargo, como indica Castles, un rasgo importante del mercado de trabajo asiático que afecta seriamente a la dinámica de los flujos. Se trata del papel que juega en este sistema la denominada "industria migratoria", ya que la mayor parte del reclutamiento de trabajadores se realiza a través de agentes y de los denominados labor brokers. Gobiernos y empleadores han preferido tradicionalmente dejar en manos de intermediarios la organización de los flujos de trabajadores (Castles, 2001; Martin: 1996).

En los países del Golfo Pérsico las políticas de regulación migratoria han sufrido importantes cambios desde los años setenta. La demanda de mano de obra para los proyectos de infraestructura y el sector servicios de las ricas economias de los paises productores de petróleo se cubrió en un primer momento con ciudadanos musulmanes de paises vecinos como Egipto, Yemen o Jordania, pero las crecientes necesidades de los ochenta provocaron la llegada de trabajadores de más lejos, en especial, del sudeste asiático y de paises del Asia meridional. Han sido muy importantes, por ejemplo, las corrientes de trabajadores manuales y de mujeres procedentes de Pakistán, India, Sri Lanka, Indonesia o Filipinas. La mayor parte de los países exportadores de petróleo planearon un sistema de reclutamiento temporal que permitiera reducir el stock de trabajadores extranjeros una vez que fueran concluidas las infraestructuras. Sin embargo, la continuidad en la demanda ha terminado por afectar a la dinámica del establecimiento. La pauta que observamos en este sistema migratorio en los años noventa es la de una economia con una alta dependencia de la mano de obra extranjera que suele superar el $60 \%$ del total. El incremento del desempleo entre los jóvenes, la estabilización de los precios del petróleo y el deseo de evitar la dependencia de los extranjeros ha activado en estos paises, en especial, en Arabia Saudita, el desarrollo de una política de "renacionalización» o "sauditizaciónı de la mano de obra a través, por ejemplo, del incremento de los impuestos a los empresarios que contratan a trabajadores foráneos. Este programa están teniendo por el momento resultados limitados a pesar de los planes que anuncian medidas drásticas de control de la entrada y de deportación. Las causas son la reticencia de los jóvenes a trabajar en empleos poco cualificados y el menor precio del trabajo de los inmigrantes.

Israel es un caso especial en el panorama presentado hasta el momento como consecuencia de la promoción de la instalación de ciudadanos judíos de todo el mundo a partir de la aliyah o ley de retorno. Este sistema ha permitido el establecimiento de un millón de ciudadanos entre 1989 y 2001, lo que explica en buena medida el ritmo de crecimiento de la población de este pais. Muchos de estos nuevos inmigrantes son profesionales altamente cualificados procedentes de los antiguos países comunistas, que están teniendo un papel clave en el desarrollo económico y tecnológico del Estado de Israel.

A pesar de sus vínculos, las políticas de refugio pueden ser consideradas políticas públicas diferenciadas de las politicas de inmigración como consecuencia de su construcción a partir de distintos principios. Como han señalado Suhrke y Zolberg, mientras que las politicas de inmigración sirven principalmente para seguir el dictado y los intereses de los paises receptores a través de la selección de los candidatos a la inmigración y el control de los flujos no deseados, las politicas de refugio surgen de las obligaciones morales y legales que los Estados liberales tienen con la comunidad internacional (Suhrke y Zolberg, 1999). Sin embargo, este compromiso ha sido interpretado de muy diversa forma dependiendo de coyunturas políticas y geoestratégicas. A lo largo de la última década hemos asistido a reformas de gran calado en las leyes nacionales y a una interpretación restrictiva del derecho internacional en materia de asilo y refugio. Con el objetivo de reducir el número de solicitudes se han establecido, por ejemplo en Europa, procedimientos de admisión a trámite o la reducción de los beneficios sociales que pueden disfrutar los solicitantes mientras esperan la resolución. Sin embargo, aunque ha sido evidente que una abultada proporción de aquellos a quiénes se les ha denegado este estatuto han permanecido en el pais donde realizaron su solicitud, se han enfatizado acciones más 
exclusionistas que han impedido tanto el acceso de los solicitantes, como la contención de la entrada de inmigrantes laborales a través de la vía del refugio (Adelman, 1991). La estrategia de limitación del acceso puede tomar diversas formas. La más cuestionable desde una perspectiva ética y desde la vulneración del principio de non-refoulement ${ }^{17}$ es la interceptación en un territorio neutral o en una frontera y la devolución sin que se permita realizar la solicitud. Otras formas han sido el establecimiento de restricciones en la concesión de visados, la ampliación de las medidas de amparo temporal, la elaboración de una lista de Estados seguros que presumiblemente no producen refugiados o la denegación de las solicitudes de aquellos que han obtenido protección o pudieron solicitarla en un tercer pais.

\section{NOTAS}

1 Algunas definiciones adicionales de politica migratoria pueden consultarse en López Sala, A (2005a): Inmigrantes y Estados. La respuesta política ante la cuestión migratoria, Barcelona, Anthropos.

2 A través del principio denominado de preferencia comunitaria (DO C 274 de 19 de septiembre de 1996).

3 Estas tesis se mantiene en la edición de 2004 en donde se recogen algunos nuevos estudios de caso. Véase Cornelius, W; Tsuda, T; Martin, P y Hollifield, J (2004): Controlling Immigration. A Global Perspective. Stantford University Press y CCIS. San Diego.

4 Cornelius, W (2000): «Acogida ambivalente: economia, cultura y etnia en las políticas de inmigración de los Estados Unidos" en Foro Internacional. Volumen $\mathrm{XL}, \mathrm{n}^{\circ} 1$, 41-63.

5 En 1995 se inauguró, por ejemplo, un centro de investigación tecnológica para adaptar tecnología militar experimental y de inteligencia a la frontera entre México y Estados Unidos. Sobre esta cuestión véase Krauss, E y Pacheco, A (2004): En la línea. México, Plaza y Janés y Martínez, $R$ (2003): Cruzando la frontera. La crónica imparable de una familia mexicana que emigra a Estados Unidos. México, Planeta mejicana.

6 Puede traducirse literalmente como prevención a través de la persuasión Se trata de disuadir a los migrantes mediante la construcción de vallas, el incremento de la vigilancia y el aumento de las penas a los coyotes y a los irregulares. Esta estrategia se diferencia de la adoptada en décadas anteriores ya que la detención y apresamiento se realizaba anteriormente una vez que se habia cruzado la frontera.
7 Posteriormente denominada de forma más politicamente correcta como Hold the Line.

8 Que incluyó la polémica medida de construir una triple valla -la intermedia con material militar empleado en la primera Guerra del Golfo- a lo largo de 14 millas en la frontera que separa las ciudades de San Diego y Tijuana.

9 El denominado Minuteman Project (MMP) es una asociación de ciudadanos voluntarios dedicados a la vigilancia de la frontera de Arizona que lleva funcionando en el Condado de Cochise desde hace dos años y medio con el objetivo de realizar una "defensa civil del territorio". Según los datos de la organización en este tiempo han facilitado a la patrulla fronteriza la detención de 4. 609 personas y se han llevado a cabo 158 rescates "humanitarios". Dos lemas empleados en la publicidad del proyecto reflejan sus objetivos: "Americans doing the jobs our Government won't don $y$ "Operating within the law to support enforcement of the lawn. Minuteman es un término utilizado en la historiografía americana para denominar a los milicianos revolucionarios que estaban preparados para sus deberes militares "al instante». Este término fue empleado por primera vez en 1774 en el condado de Worcester, Massachussets, cuando los líderes revolucionarios intentaron reformar los regimientos militares, forzando la dimisión de los antiguos oficiales y reclutando nueva tropa. 
10 "La Proposición 187 negaba a los inmigrantes irregulares residentes en California prácticamente todas las prestaciones públicas a las que, de una forma u otra, hasta entonces tenian acceso. Entre las más importantes, quedaban denegadas la enseñanza pública tanto elemental y media como universitaria, la asistencia sanitaria pública, a excepción de la de carácter de emergencia, y otro tipo de ayudas familiares" (Rubio: 1996, 35). Véase también Bustamante, J (2001): „Proposition 187 and Operation Gatekeeper. Cases for the Sociology of International Migration and Human Rights» en Migraciones Internacionales. Vol 1, no 1, 7-34.

11 Las medidas de primera acogida y el rescate maritimo salvan vidas, pero por el momento resulta extremadamente complicado evaluar el impacto que el control fronterizo tiene en la probabilidad de muerte de los migrantes que inician el cruce. La escasez de datos se combina con el hecho de que algunos de los cuerpos de los fallecidos nunca llegan a ser recuperados, por lo que empieza a emplearse el término de "desaparecidos en frontera".

12 Las críticas que han recibido estas posiciones no se basan, en exclusiva, en la idea de que la integración es posible, sino en otros argumentos. Se cuestiona, por ejemplo, tanto la pretendida homogeneidad de las sociedad de destino, como el desarrollo de ideologias exclusionistas a partir de visiones nacionales esencialistas basadas en el mito y en un escaso conocimiento de la historia (Fernández-Armesto, 2004).

13 Las categorías incorporadas en este sistema incluye tanto a empresarios como a técnicos con ofertas de empleo (dentro del denominado Employer Nomination Sche$m e)$, profesionales liberales independientes y personas con especiales talentos o cualificaciones.

14 Similares a los empleos denominados con la letra $D$ en inglés-dirty, dangerous and difficult. Estos últimos son también los nichos laborales en los que se emplean una buena parte de las mujeres (véase Figueroa, 2004).
15 Asi se denomina a los trabajadores de ascendencia japonesa procedentes de Brasil y Perú. A partir de la Immigration and Refugee Control Act de 1990, pueden establecerse en territorio nipón descendientes de ciudadanos japoneses hasta la tercera generación. Esta categoría y sus familiares de primer grado son los únicos extranjeros que según el sistema actual pueden establecerse e incorporarse al mercado de trabajo japonés sin restricciones. Los argumentos utilizados para el reclutamiento de esta categoria de trabajadores han sido de naturaleza histórica y cultural: por un lado, la deuda que Japón contrajo hacia los países latinoamericanos que recibieron ciudadanos japoneses en un momento especialmente duro en su historia $y$, en segundo lugar, el entendimiento de los códigos culturales japoneses $y$ tener usangre" nacional, esta vez en un sentido literal.

16 También es habitual el cobro de impuestos a los trabajadores extranjeros. En Singapur donde las medidas para evitar la instalación de los extranjeros son más rígidas se prohiben incluso las visitas de familiares de trabajadores y las mujeres son sometidas a pruebas de embarazo pudiendo llegar a ser deportadas por esta razón. EI matrimonio con un ciudadano de Singapur no asegura el derecho a establecerse.

17 Principio que impide la devolución a un lugar donde pueden correr el riego de ser perseguidos.

\section{BIBLIOGRAFÍA}

Adelman, H (1991) (comp.): Refugee Policy: Canada and the United States, Toronto, Center for Refugees Studies.

York Lanes Press.

Adepoju, A (2000): "La migración internacional en el Africa subsahariana: problemas y tendencias recientes" en International Social Science Journal, $n^{\circ} 165,133-147$.

Adepoju, A (2001): «Regional Integration, Continuity and Changing Patterns of Intraregional Migration in Sub-Saharian African en Siddique, $M$ (edit.), International Migration into the $21^{\text {st }}$ century, Cheltenham, Edward Elgar, 29-50.

Alarcón, R (2000): "Skilled Immigrants and Cerebreros: Foreign-Born Engineers and Scientists in the High-Technology Industry of Silicon Valley" en Foner, $\mathrm{N}$; Rumbaut, R y Gold, S (comps.), Immigration Research for a New Century. Multidisciplinary Perspectives; Nueva York, Russell-Sage Foundation, 301-321. Alarcón, R (2001): The Search for the Best and Brightest? Skilled Immigrants in the High Technology Industry and the Role of U.S Immigration Policy, La Joya, Center for Comparative Immigration Studies. Working Paper $n^{\circ} 16$. Universidad de California-San Diego.

Alba, F (1999): "La política migratoria mexicana después de IRCA» en Estudios Demográficos y Urbanos. Vol 14, no 1,11-37.

Aleinikoff, T (2002): International Legal Norms and Migration: An analysis. International Dialogue on Migration, $n^{\circ} 3$, Ginebra, IOM.

Andreas, P (1998): "The U.S Immigration Control Offensive: Constructing an Image of Order on the Southwest Bordern en Suárez Orozco, M (edit.) Crossing: Mexican Immigration in Interdisciplinary Perspectives, Boston, The David Rockefeller Center Series on Latin American Studies. Harvard University, 343-361.

Andreas, P (2000): Border Games. Policing the U.S-Mexico Divide, Ithaca, Cornell University Press. 
Arango, J y Sandell, R (2004): Inmigración: prioridades para una nueva política española, Madrid, Instituto Universitario Ortega y Gasset y Real Instituto Elcano.

Boswell, C (2003a): European Migration Policies in Flux. Changing Pattern of Inclusión and Exclusión, Londres, The Royal Institute of International Affairs. Blackwell.

Boswell, C (2003b): "The external dimension of EU immigration and asylum policy" en International Affairs. Vol 79, $n^{\circ} 3,619-638$.

Bustamante, J (2001): «Proposition 187 and Operation Gatekeeper. Cases for the Sociology of International Migration and Human Rights" en Migraciones Internacionales. Vol 1, no 1, 7-34.

Carens, J (1996): «Realistic and Idealistic Approaches to the Ethics of Migration" en International Migration Review. Vol 30, no 1, 156-170.

Carens, J (1997): «The Philosopher and the Policymaker: two perspectives on the ethics of immigration with special attention to the problem of restricting asylum» en Hailbronner, $\mathrm{K}$; Martin, D y Motomura, $\mathrm{H}$ (edits.), Immigration admissions: the search for workable policies in Germany and the United States, Londres, Berghahn Books, 3-50.

Carens, J (2002): «Inmigración y Justicia: ¿a quién dejamos pasar? en Isegoría", $n^{\circ} 26,5-27$.

Castles, S (1998): «New migrations in the Asia-Pacific region: a force for social and political change" en International Social Science Journal. Vol 156. Pág 215-228.

Castles, S (2001): "International Migration and the Nation State in Asia" en Siddique, M (edit.), International Migration into the $21^{\text {st }}$ century: essays in honour of Reginald Appleyard, Londres, Edward Elgar, 178-200.

Castles, S (2003): "La política internacional de la migración forzadan, en Migración y Desarrollo no 1, 74-90.

Cornelius, W; Martin, P y Hollifield, J (1995): Controlling Immigration: A global perspective, Stantford, Stantford University Press.
Cornelius, W; Tsuda, T; Martin, P y Hollifield, J (2004): Controlling Immigration. A Global Perspective, San Diego, Stantford University Press y CCIS.

Cornelius, W (2000): «Acogida ambivalente: economía, cultura y etnia en las politicas de inmigración de los Estados Unidosn en Foro Internacional. Volumen $\mathrm{XL}$, no 1, 41-63.

Douglass, M y Roberts, G (2000): Japan and Global Migration: Foreign workers and the advent of a multicultural society, Londres, Routledge.

Faist, T (2002): «Extensión du domaine de la lutte. International Migration and Security before and after September 11,2001 " en International Migration Review. Vol 36, no 1.

Fernández-Armesto, F (2004): «Estados Unidos: la esencia y el miton en Los Hispanos en Estados Unidos, Barcelona, Vanguardia Dossier, no 13.

Freeman, G (1999): "The quest for skill: a comparative analysis» en Bernstein, A y Weiner, $M$ (edits.), Migration and Refugee Policies, Londres, Pinter, 84-118.

Figueroa Saavedra, M (2004): "La situación laboral de la mujer en Japón" en Cuadernos de Relaciones Laborales. Vol 22, n०2, 167-195.

Fukuoka, Y(2000): Lives of Young Koreans in Japan, Melbourne, TransPacific Press.

Gabbacia, D (1999): «The Yellow Peril and the Chinese of Europe: Global Perspectives on Race and Labor, 1815-1930" en Lucassen, L y Lucassen, $\mathrm{J}$ (edits.), Migration, Migration History, History. Old Paradigms and New Perspectives, Berlín, Peter Lang, 177-196.

Geddes, A (2003): "Migration and the Welfare State in Europen en The Political Quarterly. Vol 74, n¹, 150-162.

Goodman, R; Peach, C; Takenaka, A y White, P (2003) (edits): Global Japan. The experience of Japan's new immigrant and overseas communities, Londres, Rouledge.

Gungwu, W (1993): "Migration and Its Enemies" en Mazlish, B y Buultjens, $\mathrm{R}$ (edits.), Conceptualizing Global History, Oxford, Westview Press, 131-151.
Hammar, T (1999): "Closing the doors to the Swedish Welfare State» en Hammar, Ty Brochmann, G (edit), Mechanisms of Immigration Control: a comparative análisis of European Regulation Policies, Oxford, Berg, 169-201.

Hammar, T (2001): «Politics of Immigration Control and Politicisation of International Migration" en Sidique, $M$ (edit), International Migration into the $21^{\text {st }}$ century, Cheltenham, Edward Elgar, 15-28.

Hugo, G (2003): Migrants and their integration: contemporary issues and implications. Paris, UNESCO.

Huntington, S (2004): Who are We? The Challenges of America's National Identity, Nueva York, Simon and Schuster.

Ingram, D (2002): «Immigration and Social Justice» en Peace Review. Vol 14, $n^{\circ}$ 4, 403-413.

IOM (1997): Trafficking in Women to Japan for Sexual Explotation. Ginebra.

Izquierdo, A; López, D y Martínez, R (2002): "Los preferidos del siglo XXI: la inmigración latinoamericana en España" en García Castaño, J y Muriel, C (edits.), La inmigración en España: contextos y alternativas, Granada, Laboratorio de Estudios Interculturales, Universidad de Granada, 237-250.

Krauss, E y Pacheco, A (2004): En la línea. México, Plaza y Janés.

López Sala, A (2003): «Politique migratoire et discrimination dans le cas espagnol" Revista Confluencias Mediterraneé, Discrinations ethniques. $n^{\circ} 48$, Paris, L'Harmattan, 137-148.

López Sala, A (2005a): Inmigrantes y Estados. La respuesta política ante la cuestión migratoria, Barcelona, Anthropos.

López Sala (2005b): "Matters of State»? Migration Policy-Making in Spain as a new political domain» en European Journal of International Migration and Ethnic Relations. Berlin,

The European Migration Centre. Institute for Comparative Social Research (en prensa).

López Sala, A (2005c): «Las políticas canadienses de inmigración: una perspectiva histórica de intervención estatal sobre los extranjeros" en Arbós, X (comp.), El federalismo canadiense. Barcelona, UAB y $A E E C$ (en prensa).

ARBOR CLXXXI 713 MAYO-JUNIO [2005] 27-39 ISSN: 0210-1963 
Martin, P (1996): "Labor contractor: a conceptual overview" en Asian and Pacific Migration Journal. Vol 5, $n^{\circ} 1,201-21$

Martin, P y Widgren, J (2002): International Migration: Facing the Challenge. Population Bulletin. Vol 57, $n^{\circ} 1$, Washington, Population Reference Bureau.

Martin, P (2003): Bordering on Control. Combating Irregular Migration in North America and Europe. IOM Migration Research Series, no 13, Ginebra, I0M.

Martinez, E y Moualhi, D (2005): "Actitudes ante las politicas de inmigración" en Torcal, M; Morales, L y Pérez-Nievas, S (edits).: España: sociedad y política en perspectiva comparada. Un análisis de la primera ola de la Encuesta Social Europea, Valencia, Tirant lo Blanch, 333-358.

Martínez, R (2003): Cruzando la frontera. La crónica imparable de una familia mexicana que emigra a Estados Unidos. México D.F, Planeta mejicana.

Massey, D (1999): «International Migration at the Dawn of the Twenty-First Century: the role of the Staten, Population and Development Review. Vol 25, n² 2, 303-322.

Massey, D y Durand, J (2003): Clandestinos, Zacatecas, Universidad Autónoma de Zacatecas. Colección América Latina y el Nuevo Orden Mundial.

Messina, A (1996): «The Not So Silent Revolution: Postwar Migration to Western Europen, en World Politics, $n^{\circ}$ 49,130-154.

Miyoshi, H (2003): «Policy problems relating to labor migration control in Japan" en Goodman, R; Peach, C; Takenaka, A y White, $P$ (edits.): Global Japan. The experience of Japan's new immigrant and overseas communities, Londres, Rouledge, 57-76.

Monar, J (1997): "Comprehensive Migration Policy. The main elements and options" en Hailbronner, K; Martin, D y Motomura, $\mathrm{H}$ (edits.), Immigration admissions: the search for workable policies in Germany and the United Status, Londres, Bergham Books, 45-77.

Piper, N (2004): «Rights of foreign workers and the politics of migration in SouthEast and East Asian, en International Migration. Vol 42, n 5, 71-97.
Rubio Marín, R (1996): «Inmigración ilegal y ciudadanía nacional: una controversia política en EEUU» en Claves de la Razón Práctica n68, 32-39.

Sartori, G (2001): La sociedad multiétnica: pluralismo, multiculturalismo y extranjeros, Madrid, Taurus.

Skeldon, R (2000): "Tendencias en la migración internacional en la Región Asia y el Pacificon en Revista Internacional de Ciencias Sociales, no 165, 118-132.

Suhrke, A y Zolberg, A (1999): "Issues in contemporary refugee policies" en Bernstein, A y Weiner, M (edits.), Migration and Refugee Policies, Londres, Pinter, 143-179.

United Nations (2000): Replacement Migration: Is it a solution to Declining and Ageing Populations? Nueva York.

Whitol de Wenden, C (1992): «Flux migratories et politiques d'inmigration européonnes" en Hommes and Migration, $\mathrm{n}^{\circ} 115,6-17$.

Whitol de Wendem, C (2000): ¿Hay que abrir las fronteras, Barcelona, Bellaterra. Colección la Biblioteca del Ciudadano.

Zapata, R (2001): «Dilemas de los Estados democrático-liberales para acomodar politicamente a la inmigración" en Anthropos no 191, 58-69.

Zincone, G (1999): «lllegality, Enlightenment and Ambiguity: A Hot Italian Recipe" en Arango, J

y Baldwin-Edwards, M. (edits,), Immigrants and the Informal Economy in Southern Europe. London, Frank Cass, 43-82.

Zolberg, A (2002): "Guarding the Gates in a World on the Moven en Social Science Research Council. Terrorism and Democratic Values. Nueva York. 